\title{
An EUDET / AIDA Pixel Beam Telescope for Detector Development
}

\author{
Hanno Perrey, \\ on behalf of the AIDA collaboration
}

\author{
1st of November 2012, \\ 2012 IEEE Nuclear Science Symposium
}


Workpackage 9.3.1 within European AIDA project aims at building a versatile beam telescope:

- able to characterize detector prototypes, satisfying the demanding user requirements in terms of cooling infrastructure, read-out speed and precision

- using state-of-the-art pixel devices: TimePix, ATLAS FE-14 and MIMOSA

- capable of LHC-speed response and time-stamping

\section{Outline}

(1) Introduction

Telescope Infrastructure by Example: EUDET

(2) Technologies

ATLAS FE-14

MIMOSA

TimePix \& TimePix Telescope

(3) Summary 


\section{The EUDET Beam Telescope Family}

- easy-to-use system with well-defined interfaces

- allows test beam studies on a short time scale

- telescope being used by many different groups

- performs well at both high and low momentum beams: track pointing resolution $\sim 2 \mu \mathrm{m}$ at $4.3 \mathrm{kHz}$ trigger rate

- Currently at SPS-H6B, running at DESY during SPS maintenance

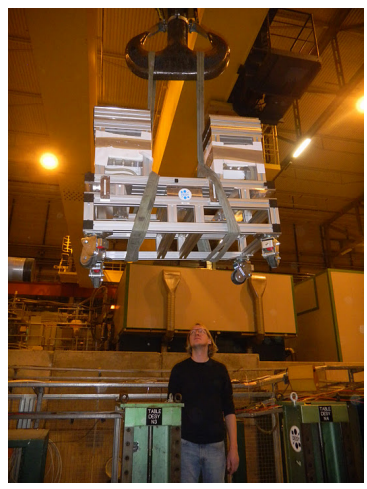

- commercial DAQ hardware $\rightarrow$ "easy" copies:

- Bonn copy (ANEMONE)

- ATLAS copy (ACONITE)

- DESY test beam (DATURA)

- ...

- Goal: same infrastructure at DESY TB and CERN SPS TB 


\section{Components of the EUDET Telescope}

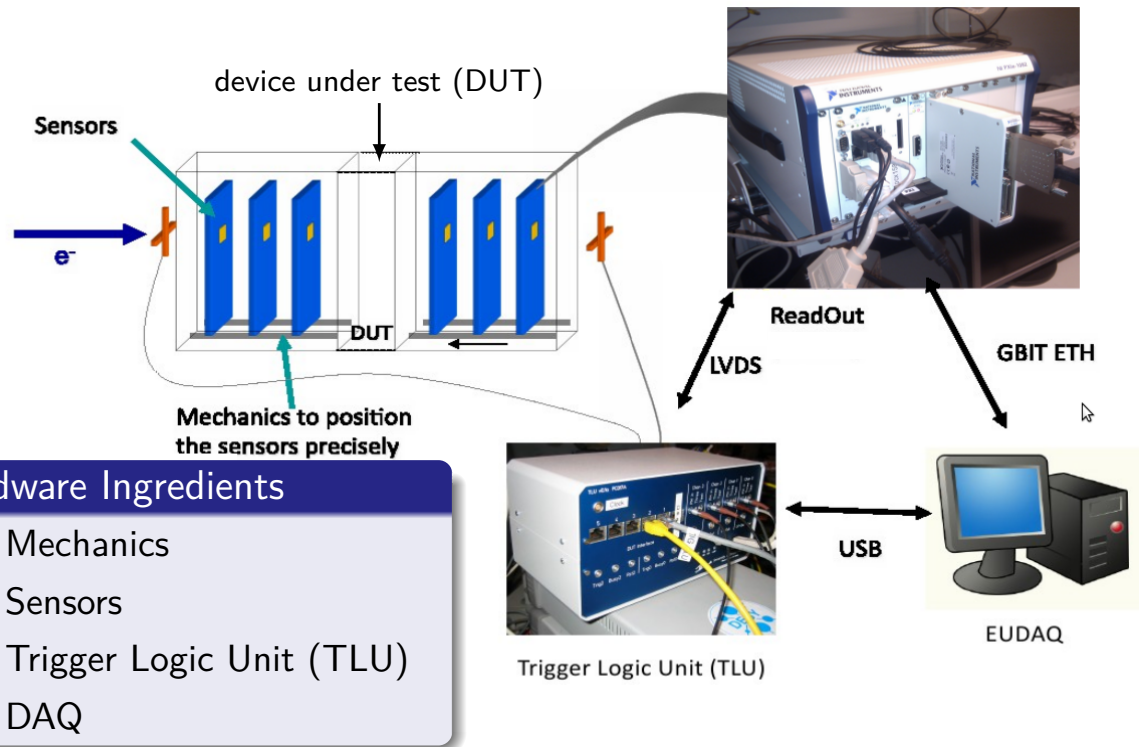




\section{Upgrade of the DAQ in 2011}

\section{DAQ (15,5 k $) \rightarrow$ Crate + Flex RIO + CPU + RAID (Option)}

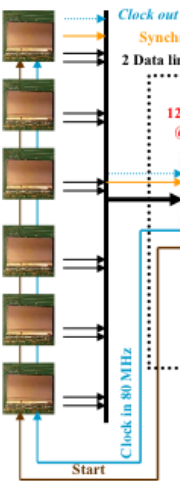

6 x Mimosa 26

\section{PXIe 1082 Crate} $\sim 3200 €$
Ethernet link

\section{Much faster \\ $\Rightarrow$ ready for the future}

EUDET SW

\section{Run ctrl \& Monitoring} $1 \mathrm{~Gb} / \mathrm{s} \rightarrow \sim 100 \mathrm{MB} / \mathrm{s}$ 


\section{The Trigger Logic Unit (TLU)}

- Gives a simple but flexible interface to trigger/timing signals

- Low cost

- Well documented ( $\rightarrow$ EUDET-Memo-2009-4)

- Many copies build by Uni Göttingen to fulfill growing demand

- Existing TLU works. Why a new one?

- Want to move to one-trigger-per-particle (instead of one trigger per telescope frame)

- Faster $\rightarrow$ needed for LHC detectors.

- Cheaper to produce TLUs for integration in home labs.

- Mini TLU prototype in preparation 


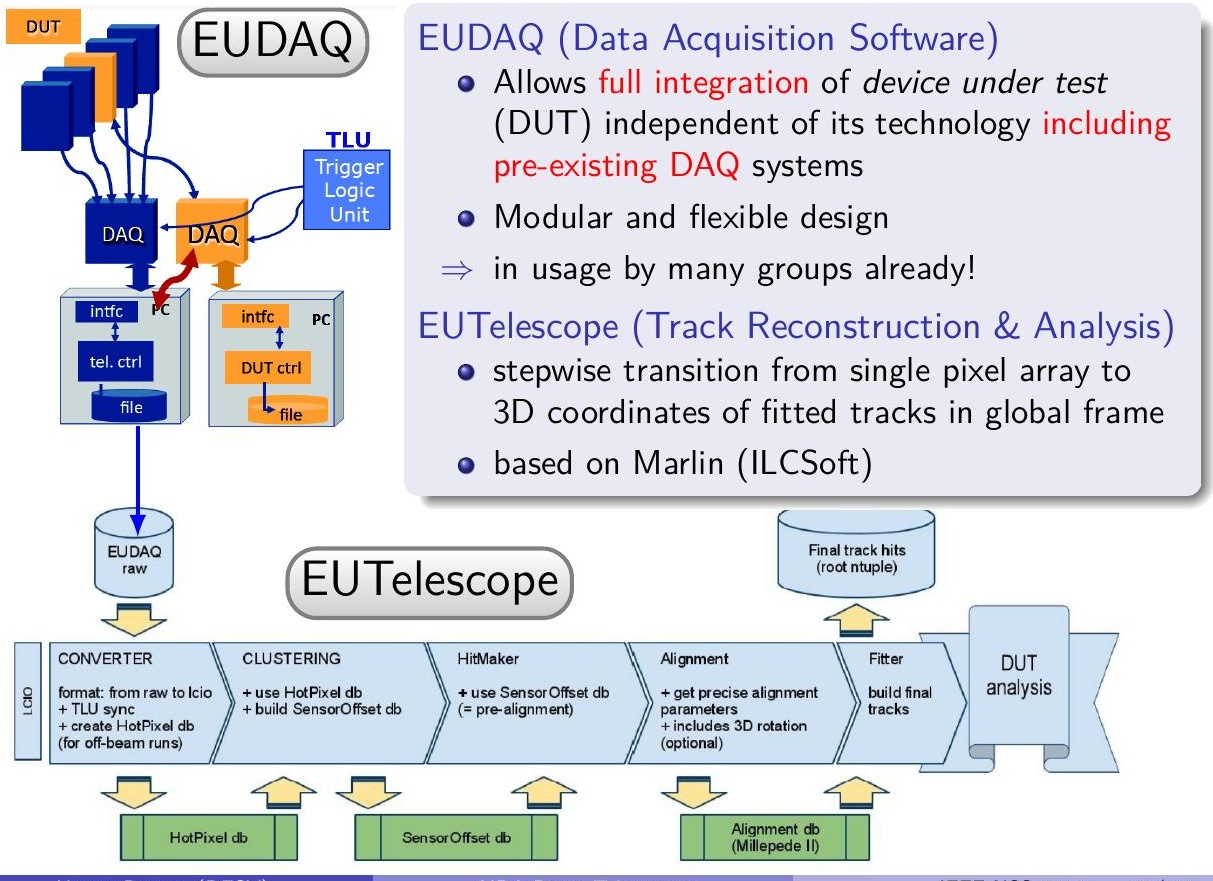




\section{One Technology: ATLAS FE-I4}

- FE-14 properties:

- $50 \mu \mathrm{m} \times 250 \mu \mathrm{m}$ pixel size

- array size: $80 \mathrm{col} \times 336$ rows

- total width: $\sim 400 \mu \mathrm{m}$

- max. trigger rate: $200 \mathrm{kHz}$

- HitOr signal for self-trigger

- FE-I4 module-based reference planes offer...

- high rate and high occupancy capability

- large area $\sim 4 \mathrm{~cm}^{2}$

- fast self-triggering in defined region of interest

- $\mathrm{R} / \mathrm{O}$ fully integrated in common infrastructure of the "AIDA telescope" framework:

- mechanics: cold operation

- DAQ-software based on the EUDAQ package

- reacts to TLU signal

- can send trigger signal to TLU
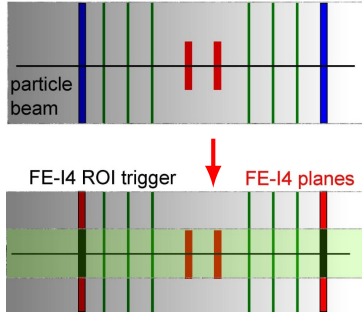


\section{ATLAS FE-I4 as Telescope Trigger}

- FE-I4 features fast HitOr:

a wired $\mathrm{OR}$ over all pixels

- each pixels HitOr can be switched on/off

$\Rightarrow$ can define region of interest

- successfully tested with EUDET telescope at DESY using MIMOSA26 reference planes

- successfully tested two, FE-14 planes at ELSA
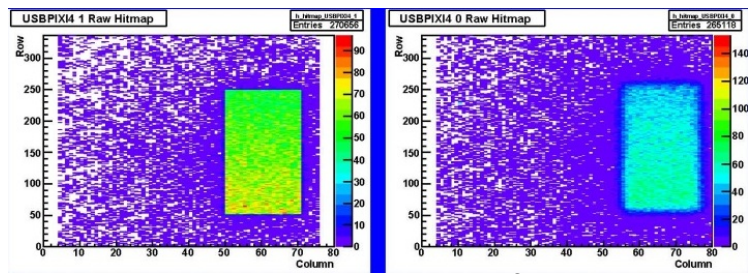

FE-I4 Hitmap, $10 \times 5 \mathrm{~mm}^{2}$ window

trigger mask
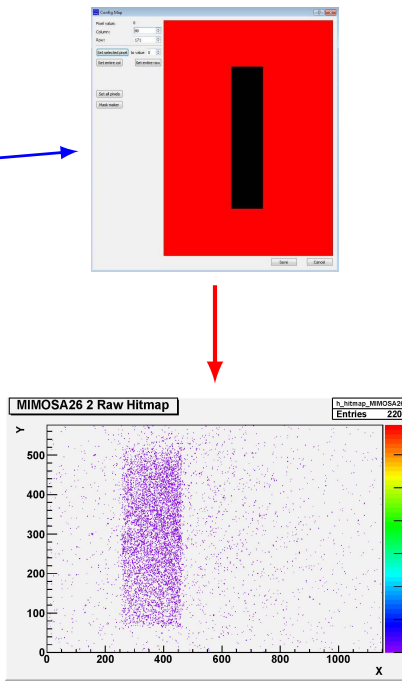

EUDET hitmap 


\section{MimaidA Sensors}

- Prominent features of Mimaida:

- very low material budget: $50 \mu \mathrm{m} \mathrm{Si}$

- high resolution: $\sim 4 \mu \mathrm{m}$

- large detection area : $4 \times 6 \mathrm{~cm}^{2}$

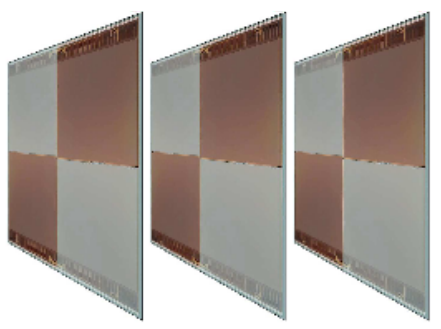

- Production of sensors in two steps:

- Start with demonstrator based on MIMOSA-28 sensors

- Replace demonstrator sensors with final sensors;

$\Rightarrow$ available for users by 2015

- relies on new $0.18 \mu \mathrm{m}$ CMOS process, currently investigated (MIMOSA-32), which provides stitching 


\section{TimePix Planes}

- TimePix readout chip on $300 \mu \mathrm{m}$ thick Si pixel sensor

- $256 \times 256$ pixels of $55 \mu \mathrm{m} \times 55 \mu \mathrm{m}$

- operating modes: counting, time of arrival, time over threshold

- global shutter signal controls when pixels are active

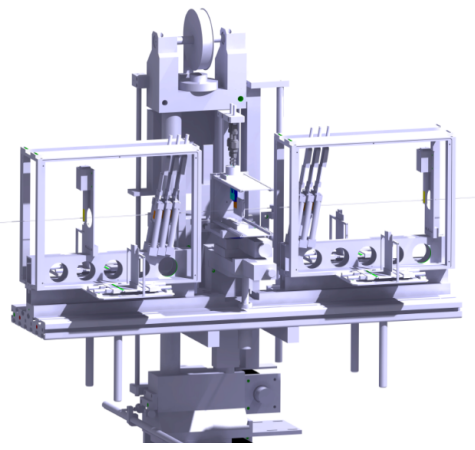

TimePix Telescope Features:

- $\sim 12 \mathrm{kHz}$ track rate

- Spatial resolution $\sim 1.5 \mu \mathrm{m}$

- Time stamping with $\sim 1 \mathrm{~ns}$ precision

- (disadvantage: material budget degrades resolution for lower energy beams)

- Telescope available to LHCb collab. and in

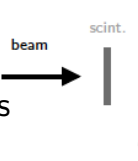
the framework of AIDA WP9.3 to external

$2 \times 4$ TimePix planes in ToT mode

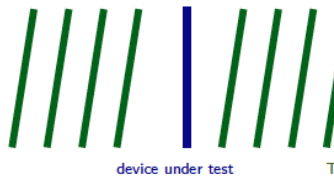

(position/rotation remotely adjustable)
TimePix plane

in ToA mode 


\section{TimePix Telescope: Integration with external users}

Effort to make external integration as simple as possible:

- DAQ systems run independently, information combined offline

- $\mathrm{CO}_{2}$ cooling, remote controlled translations and full rotations, large space in $z$ and $y$ directions provided to DUT

- External integration works with $40 \mathrm{MHz}$ style readouts without need for tagging plane.

- Proved with scintillating fibers, FE-14 with full rotations, strip detectors, etc.

- Excellent quality FE-I4 data:

TimePix/FE-14 resolution

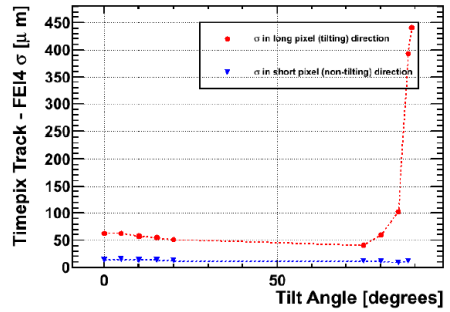

Track positions for $1,2,3,>3$ pixel FE-14 clusters
0 degrees

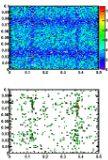

15 degrees
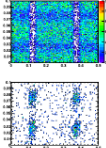

5 degrees
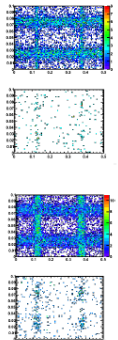
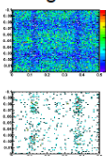

20 degrees
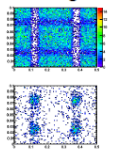
10 degrees
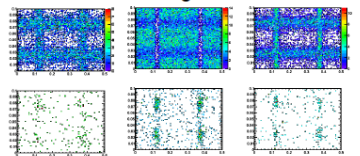

75 degrees
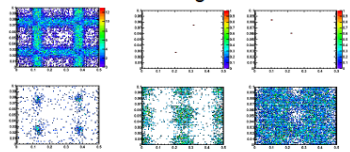


\section{Summary}

- The EUDET-family of telescopes continues to evolve and will be available at DESY and CERN in different stages most of the time

- TimePix telescope is installed at CERN (and is now a part of AIDA)

- AIDA will not provide one telescope with a lot of features but a couple of telescopes with different features!

Telescopes are available to interested users!

contact: pixel-telescopes@desy.de 